\title{
Testing the H.264 error-resilience on wireless ad-hoc networks
}

\author{
C. M. Calafate and M. P. Malumbres \\ Department of Computer Engineering (DISCA) \\ Polytechnic University of Valencia \\ Camino de Vera, S/N, Valencia, Spain \\ $\{$ calafate, mperez\}@disca.upv.es
}

\begin{abstract}
The purpose of this paper is to provide a framework to tune and evaluate the performance of the H.264 codec in $802.11 \mathrm{~b}$ wireless ad-hoc networks. The codec's error-resilience features are measured under stress conditions typical to these networks, and the most critical parameters are presented. We present solutions concerning the random packet loss problem and show how to quickly recover from packet loss bursts.
\end{abstract}

Keywords: H.264, performance, wireless, MANET, error resilience,ad-hoc

\section{INTRODUCTION}

The first video coding recommendations from IT targeted transmission of low bitrate video over ISDN, and so they were not of general purpose. The new video coding proposal H.264, which is part of an ongoing activity since 1997 named H.26L, is being developed by the Joint Video Team (JVT), an alliance formed by the former ITU-T VCEG and ISO MPEG-4 groups. Its aim is to elaborate an open standard that is not application-specific and that performs significantly better than the now available ISO MPEG-4 Part 2 [1] and ITU-T Recommendation H.263 [2] standards in terms of compression, network adaptation and error robustness.

The IEEE 802.11 family of specifications simplify the access to private networks and the Internet by developing wireless interfaces that effectively use the free electromagnetic spectrum available in most countries, providing high bandwidth at a very low cost.

IEEE's $802.11 \mathrm{~b}$ standard [3] is being increasingly used throughout corporations worldwide due to its good balance of cost, range, bandwidth and flexibility. The bandwidths set by the standard range from 1 to $11 \mathrm{Mbps}$, but other standards in the same family aim at higher bandwidths. The $802.11 \mathrm{~b}$ standard offers operation modes named Point Coordination Function (PCT) and Distributed Coordination Function (DCF). PCF is to be used in infrastructure mode, so that the Access Points are responsible for the coordination of transmissions from the various nodes. DCF, on the other hand, is a mechanism through which each node has the responsibility of sensing the medium and react to collisions. This mechanism is called CSMA/CA.

It was the DCF mode available in the $802.11 \mathrm{~b}$ standard that has pushed forward the generalized use of wireless ad-hoc networks. This networks, called MANETs (Mobile Ad-hoc NETworks) have intricacies that make them different from what can be expected in other networks, such as IP LANs, WANs and even cellular networks.

This paper focuses on the performance of H.264 in error prone environments, with special focus on its behavior in the stressing conditions usually encountered by traffic flowing inside a $802.11 \mathrm{~b}$ MANET. We also propose some guidelines on how to tune this codec for these networks based on their nature and on the achieved results. 
The strategy we followed was to start from a simple configuration where all the options from the codec were disabled by default, then study the impact on performance of each option, and finally choose the option set that offers better performance results. In a second stage the encoder was tuned using the results achieved in the previous stage in order to measure the effectiveness of the supported errorresilience options under our simulation framework.

The test model software (now renamed Joint Model) used was JM3.9a since bugs found in later versions did not allow a proper evaluation of the parameters of interest.

Concerning the structure of this paper, in the next section the available error-resilience mechanisms of H.264 will be presented. In section 3 we present the wireless channel models used and, in section 4 the simulation framework will be described. Simulation results are available in section 5 and, in section 6 concluding remarks are made showing some guidelines about future work.

\section{ERROR-RESILIENCE MECHANISMS ON H.264}

In H.264 there was a back to basics approach, were a simple design using well known block-coding schemes was used. In the design of this codec, the Video Coding Layer was separated from the Network Adaptation Layer in order to enable a modular development of each of its components. Due to its general purposed nature there is a need to include mechanisms that are able to get good performance in error prone environments such as wireless networks or the Internet.

H.264 makes available error resilience mechanisms both on the encoder and on the decoder side. In the encoder we can find several parameters that can be tuned so that a trade-off between compression rate and error resilience can be made targeting different type of problems found in heterogeneous environments.

Random intra macroblock refreshes and the insertion of intra-coded pictures (I frames) are the most commonly used methods to stop the temporal propagation of errors when no feedback channel is available. While intra frames resets the prediction process, avoiding error propagation, their use has a generally high bandwidth cost causing also severe bit rate variations. The use of random intra macroblock refreshes is more effective than I frames because not only aids to achieve CBR streams, but it can also provide better results by statistically reseting the error for each of the macroblocks. The Macroblock Line Intra Update is another robustness option where a group of blocks will be intra coded every $\mathrm{N}$ frames. It is just another form of macroblock updating.

The use of slices is another method commonly used in order to improve robustness by stopping spatial error-propagation. The macroblocks belonging to a slice can be decoded independently from other slices since no inter-slice dependencies are allowed. In our work we have used slices intensively since this mechanism is straightly related to the RTP packetization process performed by the encoder.

Another method which deserves consideration is Flexible Macroblock Ordering (FMO), whereby the sender can transmit macroblocks in non-scan order. This method, although similar to slice interleaving, provides much greater flexibility and can be tuned to be more effective in terms of error resiliency. It aims essentially at dealing with packet loss bursts.

SP slices make use of motion-compensated predictive coding to exploit temporal redundancy in the sequences, like P slices do. Unlike P slices, however, SP slice coding allows identical reconstruction of a slice even when different reference pictures are being used. They aim essentially at bit stream switching, splicing, random access, VCR functionalities and error resilience issues.

Another tool that integrates the H.264 codec is Rate Distortion Optimization. Distortion can arise due to either quantization errors and prediction from concealed blocks. If prediction does not provides good compression, intra compression for single Macroblocks is allowed. Concerning encoder tuning, it can be set to OFF for no optimization and ON if such optimization is desired. However, such values will only be optimal in the absence of errors in the network. For that reason, a third mode is available where the encoder takes into account the expected packet loss rate of the network, as well as the decoder's methods to cope with errors in order to decide weather to intra or inter code a block. See [4] for more 
details on this subject.

The constrained intra prediction option is related with the H.264 intra prediction mode, that when switched-on avoids using inter macroblock pixels to predict intra macroblocks.

Multiframe compensation prediction is another tool targeting to increase both compression performance and error resilience, since the loss of an entire reference frame will have less critical effects on later predicted frames.

Concerning the decoder, it also plays a fundamental role in error resilience since it is responsible for error concealment tasks. With that purpose it keeps a status map for macroblocks which indicates for each frame being decoded weather a certain macroblock has been correctly received, lost or already concealed. The methods used vary between intra and inter frames. For intra frames the task mainly consists on of performing a weighted pixel averaging on each lost block in order to turn it into a concealed one. For inter frames the task performed consists mainly of guessing the adequate motion vector for lost macroblocks, although intra-style methods can also be used. For a more complete description of such methods please refer to [5].

The decoder also has other tasks like handling multiple reference frames or entire frame losses.

As exposed in [4], the reference decoder for H.264 does not incorporate bit error resilience features since it increases significantly the complexity of the decoder, with only slight improvements as a result. Therefore, bit error detection and handling has to be processed externally.

\section{WIRELESS NETWORK MODELS}

Our analysis was centered on 802.11 b networks with Distributed Coordination Function. This technology uses CSMA/CA so that each unicast packet is preceeded by a RTS/CTS sequence, followed by an Acknowledge packet [3]. This process assures that the packet, when delivered, is free of bit errors. However, the Link Layer for $802.11 \mathrm{~b}$ is not connection-oriented, which means that after four failed attempts a packet is dropped. Therefore on scenarios where unicasting is used the only possible errors are related to packet losses.

When multihop ad-hoc routing protocols such as AODV[6], DSR[7], TORA[8], DSDV[9], OLSR[10] and others are used, network nodes can act as routers to other nodes, resulting in a MANET environment.

Concerning the packet loss model, we considered that there were two main types of packet losses in such an environment: random packet losses and packet loss bursts. Phenomena like collisions or errors in radio transmission and network congestions account for random packets losses. However, the effect of SNR variations perceived by nodes at near-edge distances and re-routing delays for level 3 protocols result in packet loss bursts.

In fact, long packet loss bursts can be a major source of problems for video flows inside MANETs. This problem is more evident when Hello packets are used to detect broken links. Typical Hello intervals $[6,10]$, range from 1 to 2 seconds, and so re-routing times can be has high as 6 seconds or more. Since such failures are too long to be handled even by the most versatile video codec, we recommend the use of Link Level aware protocols such as AODV-LL, DSR or TORA in order to perform re-routing tasks as soon as possible.

\section{SIMULATION FRAMEWORK}

\subsection{H.264 issues}

To evaluate the performance of the H.264 codec we used reference software JM3.9a. The evaluation was a two step process, where in a first step the codec was tuned in such a manner that all major options were turned off. The effect of each of the parameters on bit rate, SNR and encoding time were measured in order to provide enough information to bias the codec for step two, where error resilience issues were analyzed. 
Due to the absence of a bit rate control mechanism inside the codec, we tuned the codec for midrange quantization values in order to allow an external bit rate control mechanism that we developed to have the flexibility in range needed for its task. This external bit rate control mechanism consisted of a group of Perl scripts that interactively changed the quantization value for all types of frames in order to minimize the gap towards the bias value entered by the user. Such mechanism allowed us to perform our tests for step two at a constant bit rate, so that coherence and ease of interpretation was possible.

All tests were run at a frame rate of 10 frames per second, and all test sequences were in the QCIF format. We chose a mode where only P frames were active at the start of stage two (IPPP...) and since the reference software used did not support errors in B frames, their analysis was not part of our framework. Concerning the main parameters of interest after step one, the Hadamard transform, CABAC and Rate Distortion Optimization were turned ON since they presented the best results. The use of adaptive block transforms for inter and intra was set to the fully flexible mode taking into account the results available in [11].

JM3.9a reference software capabilities concerning RTP packetization and NAL encapsulation were activated in order to simplify the tasks relative to packet loss evaluation. The packetization process was set to 7 packets per frame after an initial evaluation presented in [12] and taking into account the characteristics of ad-hoc networks.

Due to the large number of results obtained, only a subset is presented here. Please refer to [12] for more results.

\subsection{Test sequences}

Concerning the test sequences used, on step one the analysis was done for both News and Foreman sequences. We considered that in step two the results achieved were not conclusive enough, and so we also included the Bus sequence in order to get results for sequences with low (News), average (Foreman) and high (Bus) levels of movement. Both News and Foreman sequences are 300 frames long, while the Bus sequence is only 150 frames long.

\subsection{Performance measurement tools}

Our evaluation of the H.264 codec was done using both PSNR measurements and a Robustness parameter $(\mathrm{R})$ that we defined. This parameter, contrarily to PSNR, does not aim at providing a measure of the quality of the sequence, but instead, offers a mean through which the ability to sustain the image quality in the presence of error is quantified.

This parameter is defined by:

$$
R=\frac{1}{N} \times \sum_{i=0}^{N} \frac{M S E_{\text {error }- \text { free }}^{i}}{M S E^{i}}, 0<\mathrm{R} \leq 1
$$

In the absence of any kind of error, the Robustness will remain at 1 . As the error-rate increases, the $\mathrm{R}$ values decrease quadratically.

\section{PERFORMANCE RESULTS}

\subsection{Random error evaluation}

On this subsection we will present the performance results of the parameters considered most relevant in random error scenarios. The purpose of these results is to allow the user to tune the codec according to the expected channel errors. It should be pointed out that all values are average values of ten consecutive simulations.

Figure 1 presents the achieved results by varying the intra frame period. As it can be seen, smaller intra frame periods perform better in scenarios with higher packet loss, achieving also better robustness. 
These results can be directly compared to those presented in figure 2 relative to random intra macroblock updates since the conclusions are similar. It should be noticed that sequences with more movement (Bus) get more benefit from frequent intra updates. In terms of quality, doing Intra Macroblock Updating improves the codec performance in medium to high motion sequences for low and moderate packet loss rates (up to 3\%). However, this improvement is based on low updating rates, avoiding to increase the final bitrate significantly. As exposed in [13], it is interesting to dynamically change the macroblock update frequency depending on the expected packet loss rate in order to maximize both quality performance and error-resilience features.

The results for the Macroblock Lines Intra Update parameter and the use of SP frames mimics the results of figures 1 and 2, therefore they were not presented here. For more information refer to [12]. In general, all of these four parameters have similar effects regarding error resilience, and tuning one of them, as the Random Intra Macroblock Updates parameter, provides satisfactory results.
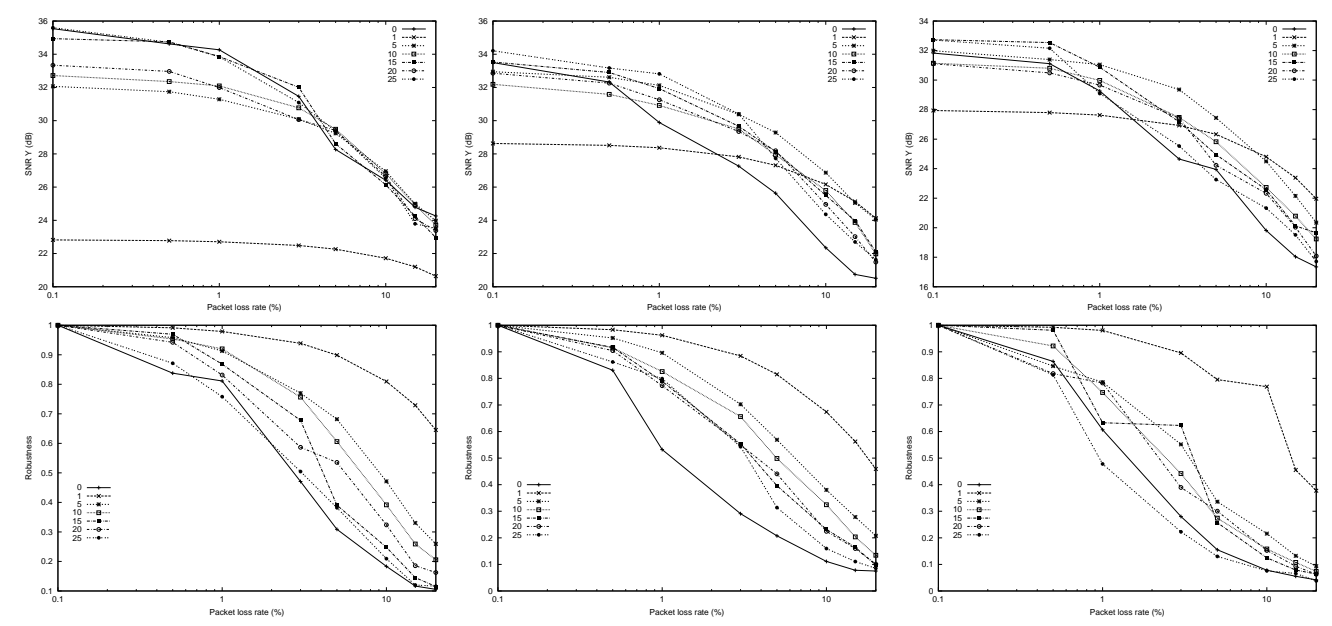

Figure 1. PSNR and Robustness results for different I frame intervals in News (left), Foreman (center) and Bus (right) sequences.
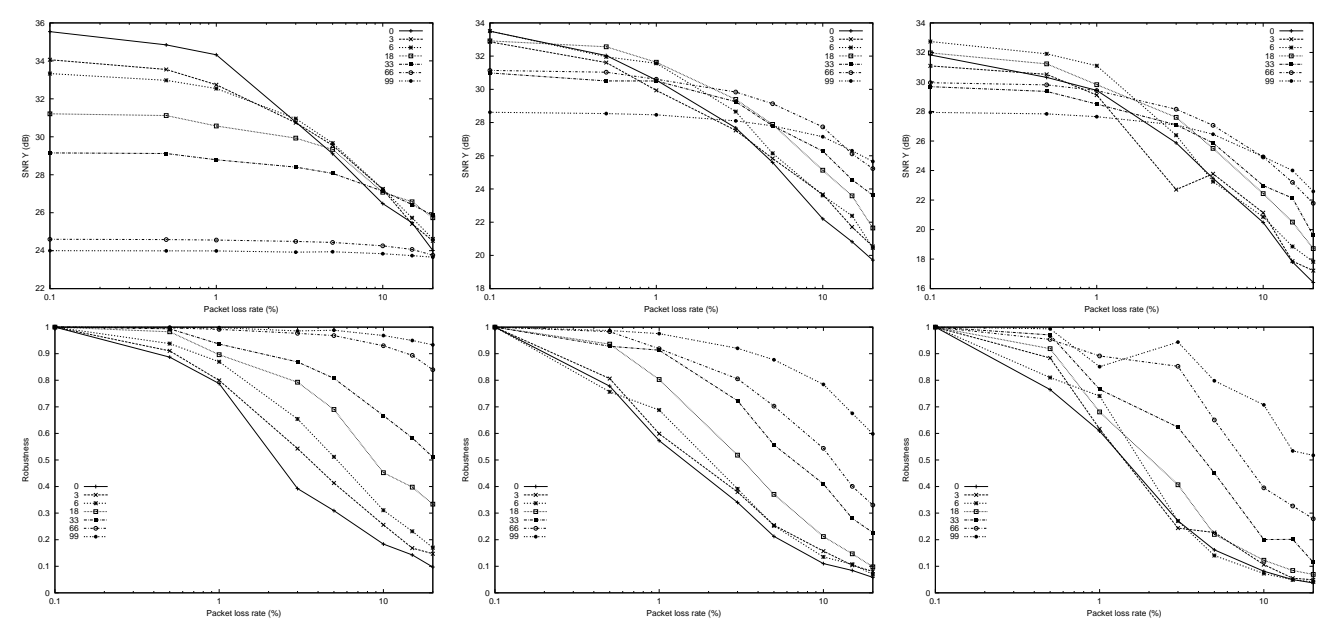

Figure 2. PSNR and Robustness results for varying Random Intra Macroblock Updates for News (left), Foreman (center) and Bus (right) sequences.

Concerning the results relative to Constrained Intra Prediction, these show that its use outperforms the original sequence when error surpasses the $4-5 \%$ to an excess than can reach $0,7 \mathrm{~dB}$. However, inferior 
results are obtained using the same parameter when the video sequence has high mobility (Bus), so we decided not to use it.

Our analysis relative to the use of multiple reference frames also doesn't provide clear results in random error scenarios, since the use of many reference frames is not always synonymous of better compression or superior error resilience, has shown in figure 3. The difference between using 4 and 5 frames of reference can reach values as high as 1,6 dB for the Bus sequence on a no-loss scenario, contrarily to what was expected.
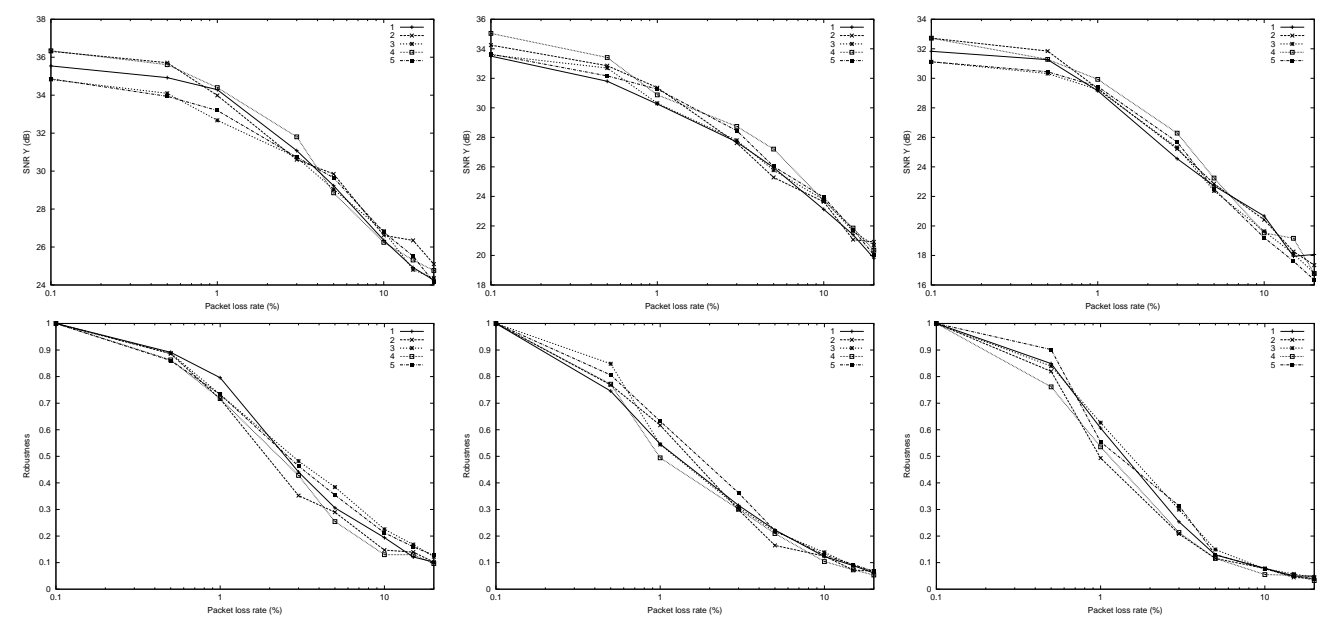

Figure 3. PSNR and Robustness results with multiple reference frames for News (left), Foreman (center) and Bus (right) sequences.

\subsection{Error burst evaluation}

For the evaluation of error bursts we used the Bus sequence, since it presents a high degree of movement. We considered that for that reason it properly stresses the codec for the evaluation being made.

We started our evaluation with small bursts affecting a single frame. Along with the regular decoding result, we also present the ones achieved by using Flexible Macroblock Ordering (FMO) mechanisms. This mechanism does macroblock rearrangement so that burst errors are distributed throughout the frame. Due to problems with the reference software, FMO tasks were performed by external software developed by the authors. Our evaluation was done using two and three groups of macroblocks; such options also belong to the H.264 framework. Other mechanisms, such as multiple reference frames, can also be used to cope with error bursts, but their evaluation was left intentionally out for future work.
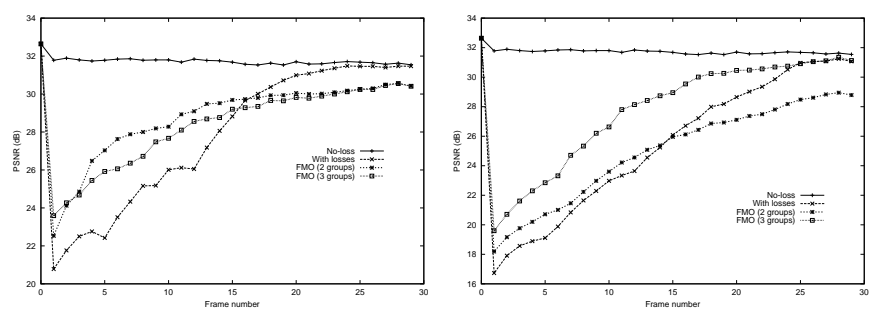

Figure 4. PSNR Recovery after error burst of $1 / 4$ (left) and $3 / 4$ (right) of frame size for 2 and 3 groups of macroblocks.

Figure 4 presents the results achieved for bursts with sizes of one-quarter and three-quarters of frame. As it can be seen, in general the use of FMO is a good option when tuning for error-burst prone networks, 
such as MANETs. Even though sometimes there are phenomena that result in difficulties to reach peak performance values on the long term, these effects can be easily canceled by intra updates, which makes FMO an attractive choice towards achieving improved error resilience.

When the error burst spreads over one or more frames, the methods used to cope with them are no longer the same. Now, the effect of mechanisms such as Random Intra Macroblock updates will be appropriate and effective, as presented in figure 5. Intra-updating just $1 / 3$ of the frames is enough to assure a quick convergence; in the worst situtation (Bus sequence) we observe that after 2 seconds the error is of just $1,4 \%$. The "\% Error" referred to is simply: $\frac{P S N R_{n o-l o s s}-P S N R_{\text {loss }}}{P S N R_{n o-l o s s}}$. The use of intra coded frames will also be effective in solving this problem, but the recovery will depend basically on the intra frame period used. It should be noticed that the reference decoder simply discards frames when no data is available, which led us to solve this problem by freezing the last available frame in order to maintain the correct number of frames on output.
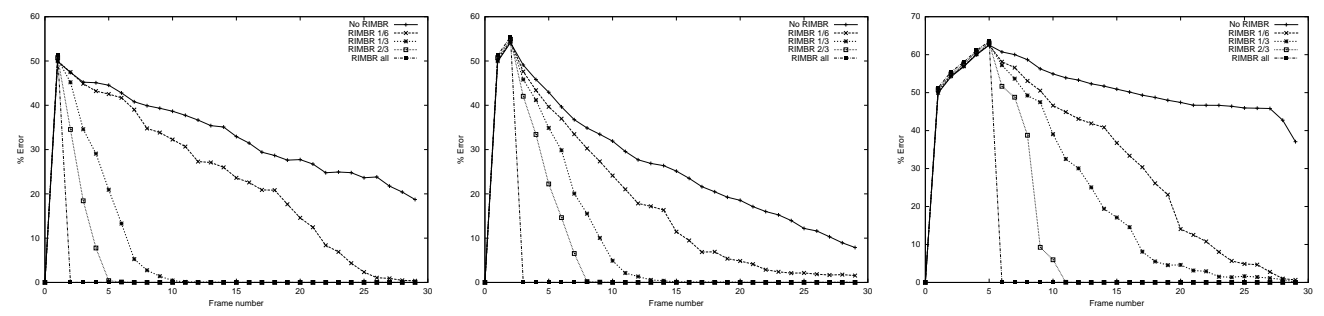

Figure 5. Error evolution after loss of 1 (left), 2 (middle) and 5 (right) frames with different level of Random Intra Macroblock updates.

\section{CONCLUSIONS AND FUTURE WORK}

In this paper we present some of the performance results obtained from our evaluation of the H.264 codec, specifically concerning the error resilience features available. Our analysis was centered on the types of error in wireless ad-hoc networks, which where modeled as random and burst packet losses. The random-loss results obtained allows tuning the encoder according to the expected packet loss rates inside the network, and show that a careful choice can increase significantly the overall PSNR of the sequence. We also present the effects of packet bursts on the quality of video and propose methods to efficiently handle these situations. Assuming a typical situation where there is a $10 \%$ packet loss in the network, tuning the Random Intra Macroblock Update to 1/3 of the frame size improves error-resilience on random and burst error situations at the cost of only a marginal increment in bitrate. Concerning the use of multiple reference frames, our study points out to the use of just two reference frames as a reasonable overall option. The FMO technique will also perform significantly well on small bursts at almost no cost.

The results presented in this paper enables tuning the H.264 codec for different network scenarios appropriately. In later works we will study its behavior in more realistic situations, using the NS[14] tool to simulate the effects of the different ad-hoc routing protocols on the video stream flow, addressing more detailed and appropriate models to radio transmission issues inherent to wireless networks. 


\section{References}

[1] "ISO/IEC IS,Coding of Audio-Visual Objects, part 2: Visual (MPEG-4)," Information Technology, November 2001.

[2] "Video coding for low bitrate communication," ITU-T Recommendation H.263, 1995.

[3] IEEE/IEC Std 802.11, Wireless LAN Medium Access Control(MAC) and Physical Layer (PHY) specifications, The Institute of Electrical and Electronics Engineers, Inc., August 1999.

[4] Thomas Stockhammer and Stephan Wenger, "Standard compliant enhancements of jvt coded video over fixed and wireless ip," 2002 International Tyrrhenian Workshop on Digital Communications (IWDC 2002), Capri (Italy), September 2002.

[5] Ye-Kui Wang, Miska Hannuksela, Viktor Varsa, Ari Hourunranta, and Moncef Gabbouj, "The error concealment feature in the h.261 test model," IEEE 2002 International Conference on Image Processing (ICIP'2002), Rochester, New York, USA, September 2002.

[6] Charles E. Perkins and Elizabeth M. Royer, "Ad hoc On-Demand Distance Vector Routing," in Proceedings of the 2nd IEEE Workshop on Mobile Computing Systems and Applications, New Orleans, LA, February 1999, pp. 90-100.

[7] David B. Johnson, David A. Maltz, Yih-Chun Hu, and Jorjeta G. Jetcheva, "The dynamic source routing protocol for mobile ad hoc networks,' Internet Draft, MANET Working Group, draft-ietfmanet-dsr-07.txt, February 2002, Work in progress.

[8] V. Park and S. Corson, "Temporally-ordered routing algorithm (TORA) version 1 - functional specification," Internet Draft, MANET Working Group, draft-ietf-manet-tora-spec-03.txt, November 2000, Work in progress.

[9] C. E. Perkins and P. Bhagwat, "Highly dynamic destination-sequenced distance-vector routing (DSDV) for mobile computers," ACM Computer Communication Review, vol. 24, no. 2, pp. 234244, October 1994.

[10] T. Clausen, P. Jacquet, A. Laouiti, P. Muhlethaler, A. Qayyum, and L. Viennot, "Optimized link state routing protocol," International Multi Topic Conference, Pakistan, 2001.

[11] Till Halbach and Mathias Wien, "Concepts and performance of next-generation video compression standardization,” 5th Nordic Signal Processing Symposium (NORSIG-2002), October 2002.

[12] Carlos Miguel Tavares Calafate, "Evaluation of the H.264 encoder (internal report)," DISCA, UPV, Spain, 2003.

[13] Thomas Stockhammer, Dimitrios Kontopodis, and Thomas Wiegand, "Rate-Distortion Optimization for H.26L Video Coding in Packet Loss Environment," 12th International Packet Video Workshop (PV 2002), Pittsburg, PY, May 2002.

[14] K. Fall and K. Varadhan, "Ns notes and documentation," The VINT project, UC Berkeley, LBL USC/ISI, and Xerox PARC, November 1997. 